\title{
FONOAUDIOLOGIA E PROMOÇÃO DA SAÚDE: RELATO DE EXPERIÊNCIA BASEADO EM VISITAS DOMICILIARES
}

\author{
Speech therapy and health promotion: \\ an experience report based on domiciliary visit
}

\author{
Bárbara Niegia Garcia de Goulart ${ }^{(1)}$, Caroline Henckel ${ }^{(2)}$, Clara Eunice Klering ${ }^{(3)}$, Maristela Martini ${ }^{(4)}$
}

\begin{abstract}
RESUMO
Objetivo: relatar a experiência de atuação fonoaudiológica para promoção da saúde baseada em visita domiciliar. Métodos: a partir da realização de visitas domiciliares e entrevistas com um morador de cada domicílio visitado foram levantadas demandas gerais ligadas ao processo de saúde-doença, bem como realizadas as orientações e encaminhamentos para os serviços de atenção básica, quando necessário. Resultados: foram visitadas 30 famílias, das quais 27 têm a mulher como principal provedora afetiva e financeira do núcleo familiar; sendo que nenhuma destas mulheres concluiu o ensino fundamental. Em média residem cinco moradores por domicílio, sendo pelo menos, duas crianças e um idoso; 10 das famílias visitadas não possuem, entre os residentes, alguém com trabalho fixo. As crianças costumam frequentar regularmente a escola. Tabagismo e alto índice de abandono dos estudos na adolescência também foram comumente referidos. Dentre as 63 crianças residentes nos domicílios visitados, os atrasos de linguagem e distúrbio fonológico consistem as alterações da comunicação humana mais comumente referidas, assim como o uso de chupeta e/ou mamadeira. Cada visita resultou em breve relato descritivo dos achados, bem como encaminhamentos e orientações realizadas. Sistematicamente foram realizadas discussões para revisão dos encaminhamentos feitos junto à equipe da unidade de saúde. Conclusões: os distúrbios de linguagem oral foram as alterações fonoaudiológicas mais comumente referidas pela população visitada, assim como a demanda por orientações em relação às funções do sistema sensório-motor-oral. Foram privilegiadas ações de promoção e educação em saúde a partir de visitas domiciliares. A continuação das atividades apresentadas prevê a avaliação da efetividade das ações desenvolvidas.
\end{abstract}

DESCRITORES: Saúde Pública; Promoção da Saúde; Educação em Saúde; Sistema Único de Saúde; Transtornos da Comunicação

\section{INTRODUÇÃO}

A saúde pública é muito rica nos seus pressupostos, fundamentados em atenção primária, medidas preventivas e educativas, além da

(1) Fonoaudióloga; Professora Adjunta da Universidade Federal do Rio Grande do Sul; Doutora em Ciências da Comunicação Humana (Fonoaudiologia) pela Universidade Federal de São Paulo.

(2) Aluna do curso de Graduação em Fonoaudiologia da Feevale, Novo Hamburgo, RS.

(3) Aluna do curso de Graduação em Fonoaudiologia da Feevale, Novo Hamburgo, RS.

(4) Aluna do curso de Graduação em Fonoaudiologia da Feevale, Novo Hamburgo, RS.

Conflito de interesses: inexistente integração entre os profissionais da saúde 1. Porém, seja por motivos político-econômicos, seja por uma cultura biomédica vigente durante dezenas de anos, até hoje, na prática, a atenção à saúde é prioritariamente voltada para a recuperação da saúde do sujeito ${ }^{2,3}$.

A Fonoaudiologia tem procurado construir seu saber, direcionando sua prática não somente no sentido do desenvolvimento tecnológico, mas também para a ampliação do conteúdo formal, responsabilidade social e política, contribuindo para a melhoria das condições de vida da população ${ }^{2,4,5}$.

O conceito de saúde está intimamente relacionado ao bem-estar biopsicossocial do indivíduo e não apenas a ausência de doenças. Desta forma, há que considerar que as habilidades e a efetividade 
da comunicação intra e inter-pessoal constituem aspectos importantes na manutenção da saúde ${ }^{4,6,7}$. A habilidade comunicativa é um elemento fundamental para a qualidade de vida e toda ação preventiva nessa área irá contribuir significativamente para promover a saúde global ${ }^{4,6,7}$.

Algumas estratégias para promoção e manutenção da saúde são apontadas por diversos autores ligados a Fonoaudiologia ou áreas afins ${ }^{2,5-9}$, dentre as quais podemos destacar as orientações para as gestantes e cuidados pré-natais ligados à prevenção e/ou diagnóstico precoce de doenças hereditárias, desenvolvimento neuropsicomotor infantil e esclarecimentos sobre fatores de risco ligados aos distúrbios da comunicação humana; prevenção e/ou detecção precoce de agravos que podem interferir na saúde e habilidades comunicativas de jovens, adultos e idosos, entre outros.

São exemplos de medidas preventivas e de promoção da saúde aquelas ligadas à atuação fonoaudiológica na promoção e orientação do aleitamento materno, o diagnóstico precoce e tratamento de desvios fonológicos e a reabilitação da comunicação de um sujeito afásico ${ }^{2,5-7}$.

A atenção básica, principalmente evidenciada pelas unidades básicas de saúde (UBS) constitui "porta de entrada" do Sistema Único de Saúde (SUS) e a oportunidade para que a imensa maioria das situações seja resolvida ou devidamente canalizada para os demais níveis de atenção previstos no sistema vigente - média e alta complexidade ${ }^{10-12}$.

A educação em saúde é o processo no qual a população participa no contexto de sua vida cotidiana e não apenas com risco de adoecer, caracteriza-se como uma prática social crítica e transformadora ${ }^{10-12}$.

A educação em saúde deve criar circunstâncias favoráveis às reflexões sobre saúde, sobre as práticas de cuidado, mudanças de comportamento potencialmente prejudiciais à saúde, aquisição de hábitos favoráveis ao bem comum e à saúde pessoal, construindo-se um dos pilares da promoção da saúde ${ }^{11,12}$.

Considerando os aspectos que norteiam a promoção da saúde em nível primário e a educação para promoção da saúde por meio de orientações à população, bem como o incentivo ao auto-cuidado, apresentamos este estudo com o objetivo de relatar a experiência de atuação fonoaudiológica para promoção da saúde baseada em visita domiciliar.

\section{MÉTODOS}

Este estudo está baseado nas vivências de três acadêmicas de Fonoaudiologia que participaram de estágio curricular obrigatório de Fonoaudiologia
Comunitária na cidade de Novo Hamburgo (RS) entre julho e dezembro de 2006.

O estágio estava vinculado a uma unidade básica de saúde que abrange aproximadamente $20 \%$ da população da cidade, ou seja, em torno de 65 mil habitantes.

No período foram visitados 30 domicílios, a partir de contato inicial com a presidente da associação de moradores do bairro, quando se decidiu visitar as famílias que tinham crianças entre zero e dez anos de idade. A proposta inicial das visitas domiciliares pretendia priorizar temas relacionados a orientações gerais sobre imunizações, higiene pessoal, importância da permanência na escola, hábitos de vida que contribuem com a promoção e manutenção da saúde, bem como questões relacionadas à comunicação humana efetiva e saudável, visto que além do interesse da fonoaudiologia, estes são pauta e constituem aspectos relacionados a políticas públicas vigentes na região.

Para nortear os trabalhos, um questionário (em anexo) com perguntas estruturadas foi utilizado para entrevista com um dos membros de cada domicílio visitado. As entrevistas e visitas foram agendadas previamente com um dos moradores de cada domicílio elegível para a visita. Na entrevista, informações ligadas ao trabalho de cada um dos moradores da casa, escolaridade, histórico de doenças crônicas ou agravos que possam trazer comprometimentos relacionados à comunicação humana tais como hipertensão arterial, acidente vascular cerebral, doenças pulmonares, frequência escolar das crianças, percepções e queixas ligadas aos serviços de saúde no município, hábitos e rotinas da família, entre outros.

Para levantamento de distúrbios fonoaudiológicos, foi utilizado protocolo padronizado e previamente validado na população deste estudo, investigando: distúrbios de fala, voz, audição e linguagem (oral e escrita) a partir de questionamento ao entrevistado sobre todos os residentes no domicílio. Para estes itens consideramos "presença de distúrbio fonoaudiológico auto-declarado" quando o sujeito respondeu positivamente para, pelo menos, um dos itens questionados.

As atividades foram desenvolvidas na Vila Kipling, território onde se encontram cerca de três mil habitantes, correspondendo a aproximadamente $4,5 \%$ da população do bairro Canudos (Novo Hamburgo, RS). Este bairro conta com duas UBS, sendo que a maior parte da população frequenta aquela onde o estágio está vinculado.

A UBS funciona das 7 às 22 horas e conta com 55 funcionários, entre médicos, técnicos em enfermagem, enfermeiro, dentistas, funcionários 
de serviços gerais, administração e recepção. São feitos, em média, 450 atendimentos por dia.

As orientações feitas pelo grupo de fonoaudiologia comunitária incluíram também rotinas e fluxos para marcação de consultas na UBS e procedimentos cirúrgicos, cadastramento no Programa Bolsa Família (desenvolvido e financiado pelo governo federal), cuidados para manutenção da saúde da criança tais como manejo do aleitamento materno e uso saudável das estruturas orofaciais para respiração, alimentação e fala, estimulação da linguagem oral, prevenção de dificuldades auditivas e medidas para preservação da saúde auditiva, além de rotinas quanto a banho e higiene geral, bem como importância do brinquedo e de freqüentar regularmente a escola para o desenvolvimento e inclusão social, incluindo orientações relacionadas a detecção precoce de dificuldades de aprendizagem. Todas as orientações foram pautadas pelo cenário encontrado quando da realização da visita domiciliar, bem como demandas trazidas pela comunidade visitada.

Quando detectada alguma alteração fonoaudiológica, a partir das entrevistas realizadas, encaminhou-se para fonoterapia e acompanhamento nas unidades de saúde da região de referência para os domicílios visitados.

Este estudo segue os pressupostos da resolução 196/96 do Conselho Nacional de Saúde e foi aprovado pelo Comitê de Ética em Pesquisa sob o protocolo n 4.06.01.07.891.

\section{RESULTADOS}

Observou-se que, em geral, as famílias visitadas foram bastante receptivas. A população aproveita a oportunidade do contato tão próximo com um profissional da saúde para esclarecer dúvidas pertinentes à saúde em geral, bem como para saber informações sobre marcações e atendimento de especialistas da UBS.

O contato com a família foi feito principalmente com mulheres, pois, comumente estas vivem sozinhas com os filhos e/ou netos e, nos casos em que contam com um companheiro, no horário em que as atividades se desenvolveram (pela manhã) a maioria dos homens estava trabalhando ou procurando emprego, conforme descreveram as entrevistadas.

No período foram visitadas 30 famílias, das quais $27(90 \%)$ tinham a mulher como principal provedora afetiva e financeira do núcleo familiar, seja a partir do gerenciamento dos valores recebidos por programas governamentais, seja por atividades realizadas no próprio lar que geram renda extra para a família, tais como prestação de serviços esporádicos para a indústria local. Nenhuma das mulheres visitadas concluiu o ensino fundamental. Em média residem cinco moradores por domicílio e 10 (33\%) das famílias visitadas não possuem em seu núcleo ninguém com trabalho fixo.

Dentre os 30 domicílios visitados, 6 (20\%) contam com pessoas que utilizam regularmente algum tipo de medicamento e em todos os domicílios visitados, quando necessário, os sujeitos recorrem à farmácia municipal para aquisição de medicamentos (pelo SUS). Nas visitas, constatouse que um dos moradores é idoso, sendo que em cada casa moram, pelo menos, duas crianças. Dentre os entrevistados, 28 (93,33\%) referiram que os moradores do domicílio fazem uso da UBS quando há doentes em seu núcleo familiar.

A grande maioria das residências visitadas ( $n=29 ; 96,7 \%$ ) dispõe de água encanada, porém, é possível observar esgoto não tratado e escoando nos pátios e vielas do bairro. Dentre os entrevistados, $28(93,33 \%)$ referiram receber recursos através do Programa Bolsa Família, mas somente seis $(20 \%)$ estavam recebendo o benefício em dia no período do estudo.

Em geral, $28(93,33 \%)$ das famílias visitadas vivem há mais de dez anos no local, mas referem que no bairro há grande rotatividade de moradores, principalmente na região com menos estrutura sanitária e mais atingida pela miséria.

As moradoras referiram que as crianças da comunidade costumam frequentar regularmente a escola e referem perceber que nos últimos dez anos houve diminuição do número de crianças na comunidade. Reportam, também, que na comunidade há muitos domicílios com tabagistas $(n=23$; $76,66 \%$ ), alto índice de abandono dos estudos na adolescência (acima de 50\%) e que o pequeno comércio que há no local se concentra na entrada da comunidade.

Dentre as 63 crianças residentes nos domicílios visitados, os atrasos de linguagem e distúrbio fonológico consistem as alterações da comunicação humana mais comumente referidas, assim como o uso de chupeta e/ou mamadeira (mais de 50\% dos sujeitos referidos como portadores de algum distúrbio fonoaudiológico), conforme descrito na tabela 1.

Para as famílias com puérperas e crianças até 2 anos foram estimulados o aleitamento materno, bem como orientações em relação a consistência dos alimentos para todos os residentes no domicílio.

Nos casos em que foram referidas ou observadas alterações fonoaudiológicas, encaminhou-se para atendimento na UBS.

As visitas iniciaram por abordagem de algum dos moradores no próprio domicílio, apresentação 
Tabela 1 - Distribuição dos Distúrbios Fonoaudiológicos e Hábitos Bucais Nocivos nas Crianças até 12 anos da População Entrevistada.

\begin{tabular}{lcc}
\hline Distúrbio Fonoaudiológico & $\mathbf{N}$ & $\%$ \\
\hline Auditivo & 2 & 3,2 \\
Alimentação & 0 & 3,2 \\
Respiração & 2 & 39,7 \\
Comunicação Oral / Fala & 25 & 4,7 \\
Escrita & 3 & $\%$ \\
\hline Hábitos Orais Nocivos & $\mathbf{N}$ & 20,6 \\
\hline Não se Aplica & 13 & 1,6 \\
Sucção Digital e ou Onicofagia & 1 & 55,5 \\
Uso de chupeta e/ou mamadeira após 24 meses de idade & 35 & 0 \\
Dorme de Boca Aberta e/ou Baba travesseiro & 0 & 0 \\
Sucção lábio inferior/ superior & 0 & 3,2 \\
Ronco ao dormir e/ou ranger dentes & 2 & 0 \\
Morde ou mantém objetos na boca & 0 & 4,7 \\
Não sabe / não respondeu & 3 & \\
\hline
\end{tabular}

rápida da dupla de visitadores e conversa guiada pelo protocolo de visita, buscando dados relacionados ao número de residentes, faixa etária, aspectos gerais de saúde e doença e, quando pertinente, indicações para retomada de acompanhamento na unidade de saúde da região, bem como reforço de informações e orientações sobre importância de manutenção do esquema vacinal, orientações para estímulo da comunicação oral em crianças e idosos com e sem queixas fonoaudiológicas, entre outros. Em média, as visitas duraram até 20 minutos, dependendo da disponibilidade do entrevistado e demais residentes presentes no domicílio.

As final de cada semana de atividades dos visitadores, uma hora de revisão e discussão de casos foi feita junto a equipe da unidade de saúde a fim de trocar informações, revisar encaminhamentos e procedimentos indicados para cada caso ou situação encontrada no núcleo familiar.

\section{DISCUSSÃO}

O breve relato dos dados advindos das visitas domiciliares e da experiência de orientação para promoção da saúde no domicílio, a partir da inserção de um grupo de fonoaudiologia na atenção básica abrange não só o encaminhamento para atendimento das alterações da saúde de maior ocorrência na população, mas atua também e, principalmente, na promoção da saúde e na prevenção de co-morbidades ${ }^{12-15}$.
Questões ligadas ao aleitamento materno e orientações para estimulação da linguagem requerem pouca complexidade de atendimento, não são necessários equipamentos sofisticados ou específicos para promover a troca de orientações e informações com gestantes, e além de atingir uma grande faixa da população - as gestantes e seus futuros bebês ${ }^{13-15}$. O trabalho realizado com gestantes é, também, uma das possibilidades de aproximação do fonoaudiólogo com a comunidade ${ }^{13,14}$. As orientações relacionadas ao aleitamento materno, prevenção de hábitos orais de sucção não nutritiva e estimulação da comunicação oral foram baseados em evidências de diversos estudos publicados na área ${ }^{13,18-27}$.

Além disso, o conhecimento mais detalhado das características da população visitada e seu entorno permite um planejamento de ações potencialmente mais efetivas e diretamente relacionadas ao panorama encontrado quando das visitas nos domicílios. A inserção do profissional na residência dos potenciais pacientes possibilita um diagnóstico da situação vivida no cotidiano possivelmente mais próximo das vivências da comunidade e permite que as orientações para mudança de hábitos que visem a melhora da comunicação ou mesmo que potencializem as habilidades de comunicação e alimentação, no contexto da atuação da fonoaudiologia, sejam demonstradas e vivenciadas pelo pelo sujeito e seus familiares em um ambiente que lhe é mais familiar, a própria residência.

A atuação fonoaudiológica junto a atenção básica relatada neste manuscrito foi pautada pelo 
desenvolvimento ações de promoção da saúde da família e da comunidade, bem como prevenir doenças e outros agravos, sem deixar de lado as ações de tratamento e reabilitação, principalmente considerando que o município no qual as atividades relatadas foram desenvolvidas não conta com equipes de saúde da família, tampouco com agentes comunitários de saúde ${ }^{13-16}$. A educação popular é um dos instrumentos metodológicos fundamental para o, fortalecimento do SUS no sentido que as pessoas assumam maior controle de sua própria saúde e de suas vidas, em que a racionalidade do modelo biomédico dominante seja transformada no cotidiano de suas práticas ${ }^{10-14}$.

O trabalho de educação para promoção da saúde constitui importante ferramenta para a redução de co-morbidades e identificação precoce de distúrbios fonoaudiológicos, bem como contribui para a ampliação da disseminação de dados e informações em relação aos direitos e deveres do usuário no SUS, colaborando para que a racionalização da busca por atenção em serviços de urgência e, por consequência, ampliando a possibilidade de acesso aos cuidados médicos que são priorizados no serviço de atenção básica 2,5,7,11-17.

Desta forma, o trabalhado desenvolvido na comunidade, brevemente relatado neste manuscrito detalha diversas etapas da atuação da fonoaudiologia por meio de visitas domiciliares, buscando contribuir com a socialização de experiências no contexto da diversidade de situações encontradas na sociedade brasileira em relação ao sistema de saúde.

\section{CONCLUSÃO}

As características da população visitada e a distribuição dos distúrbios fonoaudiológicos nas crianças até 10 anos de idade ocorreram a semeIhança de outros estudos da área, sendo que os distúrbios de linguagem e fala foram as alterações fonoaudiológicos mais prevalentes, assim como o uso de chupeta e/ou mamadeira após os 24 meses de idade.

Cabe destacar a prevalência acentuada de tabagismo na população visitada, além de importante índice de abandono dos estudos por adolescentes.

A atuação fonoaudiológica a partir de visitas domiciliares constituem importante ferramenta para a promoção da saúde e, quando da participação de alunos de graduação, permitem ao acadêmico a prática de ações voltadas para a saúde coletiva, durante sua formação profissional, contribuindo para a conscientização de que a prevenção, de um modo geral, é também uma importante responsabilidade profissional, além de reforçar a possibilidade de atuação do fonoaudiólogo baseada na realidade social vivenciada pela comunidade que atende.

O trabalho desenvolvido esclareceu a população da região sobre questões ligadas às rotinas da UBS e do hospital municipal, quando necessário. Além disso, incluiu ações de prevenção em Fonoaudiologia.

É necessário que os profissionais reestruturem suas ações, deixando de centrar esforços somente em atendimentos clínicos individuais, não só fonoaudiológicos, mas nas diversas especialidades ligadas à atenção à saúde, principalmente no que concerne à atenção básica.

A continuação das atividades apresentadas prevê a avaliação da efetividade das ações desenvolvidas, verificando se as orientações e indicações feitas por ocasião das visitas domiciliares proporcionaram mudanças nas rotinas dos sujeitos visitados, bem como ampliaram seu acesso aos serviços de saúde pública na região. 


\section{ABSTRACT}

Purpose: to report the experience of SLP intervention for health promotion based on domiciliary visits. Methods: from conducting domiciliary visits and interviews with a local inhabitant of each visited home we raised general demands in the process of health and illness, as well as the counseling and referrals for primary care services whenever needed. Results: 30 families were visited, of which 27 have women as the main emotional and financial provider of the family, and none of these women completed primary school. On average there are five residents per household, with at least 2 children and an elderly, ten visited families do not have one permanent job. Children usually attend school regularly. Smoking and high rate of dropouts in adolescence were also commonly mentioned. Among the 63 children living in the visited homes, delays in language and phonological disorders consist of the most commonly mentioned alterations in human communication, as well as the use of a pacifier and / or bottle. Each visit resulted in a brief descriptive report on the findings, as well as referrals and advice were given. Systematical discussions were held in order to review the referrals made by the basic health unit team. Conclusions: the oral speech therapy related alterations were the most commonly referred to by the visited population, as well as the demand for guidance on the sensorymotor-oral functions. Focus has been place on health promotion and education based on domiciliary visits. The effectiveness of the actions performed should be evaluated.

KEYWORDS: Public Health; Health Promotion; Health Education; Single Health System; Communication Disorders

\section{REFERÊNCIAS}

1. Campos GWS. O SUS entre a tradição dos sistemas nacionais e o modo liberal-privado para organizar o cuidado à saúde. Cienc Saúde Coletiva 2007; 12(supl):1865-74.

2. Goulart BNG, Chiari BM. Humanização das práticas do profissional de saúde: contribuições para reflexão. Rev Ciência Saúde Coletiva [periódico online] 2007; Disponível em: URL: http:// www.abrasco.org.br/cienciaesaudecoletiva/artigos/ artigo_int.php?id_artigo=960

3. Vieira GO, Silva IR, Almeida JAG, Cabral VA. Hábitos alimentares de crianças menores de um ano amamentadas e não amamentadas. J Pediatr. 2004; 80(5):411-6.

4. Goulart BNG, Chiari BM. Testes de rastreamento $x$ testes de diagnóstico: atualidades no contexto da atuação fonoaudiológica. Pró-Fono. 2007; 19(2):223-32.

5. Lenz AJ, Gernhardt A, Goulart BNG, Zimmer F, Rocha JG, Vilanova JR, et al. Acolhimento, humanização e fonoaudiologia: relato de experiência em unidade básica de saúde de Novo Hamburgo. Bol Saúde. 2006; 20(2):59-69.

6. Goulart BNG, Chiari BM. Avaliação clínica fonoaudiológica, integralidade e humanização: perspectivas gerais e contribuições para reflexão. Rev Soc Bras Fonoaudiol. 2007; 12(4):335-40.
7. Goulart BNG, Chiari BM. Construção e aplicação de indicadores de saúde na perspectiva fonoaudiológica: contribuições para reflexão. Rev Soc Bras Fonoaudiol. 2006; 11(3):194-204.

8. Aciole GG, Brunelli E. Pedagogical dimensions for the promotion of citizenship within social control. Interface. 2007; 11(23):409-26.

9. Benevides R, Passos E. A humanização como dimensão pública das políticas de saúde. Cienc Saúde Coletiva. 2005; 10(3):561-71.

10. Trevisan LN, Junqueira LAP. Construindo o "Pacto de Gestão" no SUS: da descentralização tutelada à gestão em rede. Cienc Saúde Coletiva 2007; 12(4):893-902.

11. Barroso MGT, Vieira NFC, Varela ZMV. Ensino de educação em saúde, interdisciplinaridade e políticas públicas. Rev Bras Prom Saúde 2006; 19(3):182-7.

12. Vasconcelos EM. Educação Popular: de uma prática alternativa a uma estratégia de gestão participativa das políticas de saúde. Physis. 2004; 14(1):67-83.

13. Santana MCCP, Goulart BNG, Chiari BM, Melo AM, Silva EHAA. Aleitamento materno em prematuros: atuação fonoaudiológica baseada nos pressupostos da educação para a promoção da saúde. Rev Cienc Saúde Coletiva. [periódico on line] 2008; [acesso em 16 nov 2009] Disponível em: URL: 
http://www.abrasco.org.br/cienciaesaudecoletiva/ artigos/artigo_int.php?id_artigo $=2342$

14. Goulart BNG, Chiari BM. Avaliação clínica fonoaudiológica, integralidade e humanização: perspectivas gerais e contribuições para reflexão. Rev Soc Bras Fonoaudiol. 2007; 12(4):335-40.

15. Santana MCCP, Brandão KKCP, Goulart BNG, Chiari BM. Fonoaudiologia e saúde do trabalhador: vigilância é informação para a ação! Rev. CEFAC. 2009; 11(3):522-8.

16. Goulart BNG, Algayer AR. Características de um grupo de usuários do Programa de Saúde da Família na cidade de Campo Bom (RS), Brasil em 2006. Rev Cienc Saúde Coletiva 2009; 14 (supl 1): 1379-1384.

17. Chiari BM, Goulart BNG. The role of research methodology in the rational use of technology in monitoring and preventing communication disorders. An Acad Bras Ciênc. 2009; 81(3):497-502.

18. Goulart BNG, Chiari BM. Prevalência de desordens de fala em escolares e fatores associados. Rev Saúde Públ. 2007; 41(5):726-31.

19. Rea MF. O pediatra e a amamentação exclusiva. J Pediatr. 2003; 79(6):479-80.

20. Voi Trawitzki LV, Lima WTA, Melchior MO, Grechi TH, Valera FCP. Aleitamento e hábitos orais deletérios em respiradores orais e nasais. Rev Bras Otorrinolaringol. 2005; 71(6):747-51.
21. Percegani N, Araújo RMA, Silva MMS, Euclydes MP, Tinoco ALA. Conhecimento sobre o aleitamento materno de puérperas em dois hospitais de Viçosa, MG. Rev Nutrição. 2002; 15(1):10-2.

22. Lejarraga $H$, Menendez AM, Menzano E, Guerra L, Biancato S, Pianelli P, et al. Screening for developmental problems at primary care level: a field programme in San Isidro, Argentina. Paediatr Perinat Epidemiol. 2008; 22(2):180-7.

23. Andrade SA, Santos DN, Bastos AC, Pedromônico MRM, Almeida-Filho N, Barreto ML. Ambiente familiar e desenvolvimento cognitivo infantil: uma abordagem epidemiológica. Rev Saúde Públ. 2005; 39(4):606-11.

24. Sachse S, Von Suchodoletz W. Early identification of language delay by direct language assessment or parent report? J Dev Behav Pediatr. 2008; 29(1):34-41.

25. Tosato JP, Biasotto-Gonzalez DA, Gonzalez TO. Presença de desconforto na articulação temporomandibular relacionado ao uso da chupeta. Rev Bras Otorrinolaringol. 2005; 71(3):365-8.

26. Cavassani VGS, Ribeiro SG, Nemr NK, Greco AM, Köhle J, Lehn CN. Hábitos orais de sucção: estudo piloto em população de baixa renda. Rev Bras Otorrinolaringol. 2003; 69(1):106-10.

27. Maciel CTV, Leite ICG. Aspectos etiológicos da mordida aberta anterior e suas implicações nas funções orofaciais. Pró-Fono. 2005; 17(3):293-302.

DOI: 10.1590/S1516-18462010005000095

RECEBIDO EM: 26/10/2009

ACEITO EM: 15/03/2010

Endereço para correspondência:

Bárbara Niegia Garcia de Goulart

Universidade Federal do Rio Grande do Sul

Rua Ramiro Barcelos, 2600 sala 211

Porto Alegre - RS

CEP: 90035-003

E-mail: bgoulart@ via-rs.net 


\section{PROTOCOLO DE ENTREVISTA DOMICILIAR}

\section{Dados de Identificação}

Nome do Informante:

Data de nascimento: 1

Idade: anos meses

Sexo: (1) masculino (2) feminino

Mora com: (1) pais e irmãos

(3) mãe e irmãos

(5) outros: quem
(2) pai e irmãos
(4) avós
(6) sozinho

Residentes no domicilio trabalham: (1) sim $\quad$ (2) não

$\begin{array}{lll}\text { (3) somente homem } & \text { (4) somente mulher }\end{array}$

\begin{tabular}{|l|l|l|l|l|}
\hline \multicolumn{1}{|c|}{ Nome } & Idade & Sexo & Atividade (escola - série) & Vacinas \\
\hline 1. & & & & \\
\hline 2. & & & & \\
\hline 3. & & & & \\
\hline 4. & & & & \\
\hline 5. & & & & \\
\hline 6. & & & & \\
\hline 7. & & & & \\
\hline 8. & & & & \\
\hline 9. & & & & \\
\hline
\end{tabular}

Sexo 1=masculino 2=feminino; Atividade NSA=não se aplica; número corresponde anos de estudo ou indica tipo de atividade (indústria, comércio, serviços autônomos variados); Vacinas $1=e m$ dia $2=e$ squema incompleto $3=$ não se aplica 4= não sabe informar, carteira de vacinação extraviada

Dados relativos a cada um dos moradores do domicílio:

Uso do posto: ( 1 ) Sim ～( 2 ) Não (quem? Por quên)

Fumantes na residência: ( )1. Sim ( ) 2. Não

Dificuldades Auditivas: ( )1. Sim $\quad$ ( ) 2. Não $\quad$ ( ) 3. Não sabe informar

Dificuldades de Alimentação : ( )1. Sim $\quad$ ( ) 2. Não $\quad$ ( ) 3. Não sabe informar

Dificuldades de Respiração: ( 1 ) Sim $\quad$ ( 2 ) Não( ) $\quad$ 3. Não sabe informar

Pessoas com distúrbio de comunicação: ( 1 ) Sim $\quad$ ( 2 ) Não

Qual? Especifique:

Histórico atendimento Fonoaudiológico: ( 1 ) Sim $\quad$ ( 2 ) Não $\quad$ ( ) 3. Não sabe informar

Encaminhado por:

Situação Atual:

GESTANTES na casa? ( 1 ) Sim

Pré- Natal: (1) Sim (2) Não

( 2 ) Não

Local

Freqüência:

\section{Hábitos e Sintomas Sistema Sensório Motor Oral:}

(0) Não se aplica

(1) suga algum dedo

(2) roe unhas

(3) apóia o queixo com as mãos

(4) ronca

(5) range os dentes

(6) possui dificuldade com deglutição/fala
(7) suga o lábio superior/inferior

(8) morde ou mantém objetos na boca

(9) dorme de boca aberta

(10) baba no travesseiro

(11)aperta os dentes

(12) dor na ATM D/E

(13) nenhuma das alternativas

(14) Não sabe informar

ORIENTAÇÕES / ENCAMINHAMENTOS 